\title{
The influence of an opioid on the course of reparative processes
}

\author{
Lesya Mateshuk-Vatseba, Ivan Diskovskyi ${ }^{*}$
}

Department of General Anatomy, Danylo Halytskyi Lviv National Medical University, Pekarska Street, 69, 79-010 Lviv, Ukraine

\begin{tabular}{l}
\hline ARTICLE INFO \\
\hline Received 08 January 2015 \\
Accepted 24 March 2015 \\
\hline
\end{tabular}

\section{Keywords:}

skin,

animal model, reparative process, opioid.

\begin{abstract}
The objective of our work is to examine the influence of prolonged administration of an opioid, on the course of the reparative process of a multiple post-injection wound inflicted upon a white rat's skin, doing so by way of using light microscopy. The studies were carried out on 24 mature white male rats, aged 4.5-7.5 months, with weights of 130-150 g. For histological examination, the skin sections were stained with hematoxylin and eosin. The preparations were studied and photographed at the magnification of ob.x8, ey.x15 and ob.x40, ey.x10. The «Aver Media» computer system was employed for micropreparations photography. The set levels of light microscopy reveal that the administration of an opioid for a 2 week period does not affect the reparative process of a multiple post-injection wound. Moreover, in most wound canals, the formation of a complete regenerate can be seen. However, after 4 weeks of nalbufin administration, the processes of wound healing slowed and were complicated by the formation of microabscesses. After 6 weeks of opioid administration, destructive changes in the skin were detected. These, in turn, led to the incomplete regenerate formation in the multiple postinjection wound. The experimental results, hence, show the negative effect of prolonged administration of opioid on the reparative processes in the skin.
\end{abstract}

\section{INTRODUCTION.}

With regard to patients administered or who self-administer opioids and opiates, the problems that come about in the course of reparative processes remain of great importance in modern medicine [15,17]. In scientific sources, there are few publications concerning the impact of such drugs on reparative processes. Moreover, what data exist, are usually descriptive, highlight only a few fragments of this new problem and are often contradictory. Regular opioid drug use destroys the body's immune system. The weakening of the immune system is manifested in disturbances of the intracellular defense system, as well as the skin and mucosal barriers [6]. According to P.D. Brown et al. [3], subcutaneous and intramuscular injections remain the main risk factors for skin infections in such patients. The constant increase in the number of individuals self-injecting opioid drugs has definitely led to an increase in the number of cases of myonecrosis [2,8]. Myonecrosis remains one of the most serious manifestations of infections of skin and soft tissues. Indeed, the number of cases of myonecrosis in

\begin{tabular}{l} 
^Corresponding author \\
e-mail: diskovskuy@gmail.com \\
tel.:+380984412927 \\
\hline
\end{tabular}

younger persons self-injecting opioid drugs, accompanied by toxic shock syndrome, has increased in the last 10 years [5].

The above-mentioned demonstrates the need for adequate morphological and clinical studies. Thus, the purpose of our study was to investigate the influence of prolonged administration of an opioid on the course of the reparative processes involved in the healing of a multiple post-injection wound inflicted upon a white rat's skin, and through using light microscopy.

\section{MATERIALS AND METHODS}

The investigation was carried out on 24 mature white male rats, aged 4.5-7.5 months, with weights of $130-150 \mathrm{~g}$.

The experimental animals were placed within 3 groups. In the first group (5 rats), the research involved ascertaining the course of reparative processes after 2 weeks of nalbufin administration, the multiple post-injection wound being in the region of the lateral thigh. In the second series of experiments ( 5 rats), the healing of a multiple post-injection wound was investigated after 4 weeks. In the third series of experiments (5 rats), the peculiarities of the reparative processes were to be established after 6 weeks of nalbufin 
administration. The control incorporated 9 white rats, who were injected with a saline solution.

The research material consisted of histological preparations of the skin of these rat's lateral thigh surface. For histological study, the skin sections were stained with hematoxylin and eosin. The preparations were then studied and photographed at the magnifications of ob.x8, ey.x15 and ob.x40, ey.x10. The «Aver Media» computer system was used for the photography of these micropreparations.

Administration of nalbufin was conducted intramuscularly as follows: I week $-8 \mathrm{mg} / \mathrm{kg}$, II week $-15 \mathrm{mg} / \mathrm{kg}$, III week - $20 \mathrm{mg} / \mathrm{kg}$, IV week - $25 \mathrm{mg} / \mathrm{kg}$, V week - $30 \mathrm{mg} /$ $\mathrm{kg}$, VI week - $35 \mathrm{mg} / \mathrm{kg}$ [11].

All animals were kept in a vivarium within the Danylo Halytsky Lviv National Medical University. The experiments were carried out in accordance with the principles set out by the European Convention for the protection of vertebrate animals used for experimental and other scientific purposes (Strasbourg, 1986), the Directive of European Council 86/609/EES (1986), and the Law of Ukraine № 3447 - IV "About the protection of animals against cruel treatment" general ethical principles of experiments on animals, and approved by the First National Congress of Ukraine on Bioethics (2001).

Furthermore, all experiments were carried out according to the National Institute of Health Guidelines for the care and use of laboratory animals, as well as the European Council Directive on 24 November 1986 for Care and Use of Laboratory Animals (86/609/EEC), and approved by the Local Ethics Committee.

\section{RESULTS}

After 2 weeks of nalbufin administration, using light microscopy, it was found that the reparative process of the multiple post-injection wound, as well as in the control, had all phases characteristic for suitable healing processes. Some wound defects were, however, seen in the state of distinct inflammatory infiltration and vascular reaction. Intensive cell proliferation, angiogenesis and vessels overfilled with blood were also detected. Moreover, skin tissues surrounding the wound defect were infiltrated with leukocytes and macrophages, with many fibroblasts. Significantly reduced inflammatory infiltration, signs of a productive reaction with predominance of proliferative fibroblasts in infiltrate were also observed in some wound defects. Most wound defects were completely closed and the regenerate was complete. This is confirmed by the restoration of hair follicles, sweat and sebaceous glands (Fig. 1). The main components of the skin were properly structured.

After 4 weeks of nalbufin administration, the wound healing slowed down, and was sometimes complicated by the formation of micro-abscesses. A large number of capillaries of granulation tissue, diffuse slightly marked acute inflammatory infiltrate, as well as degenerative and atrophic changes in the striated muscle fibers, were detected in the wound canals (Fig. 2). Of note, in some places there was swelling and fibrosis of the reticular dermis (Fig. 3). Only a few wound defects were closed and the regenerate complete. In most cases, the remodeling phase was not detected, some wound defects were filled with rough connective regenerate and the epidermis was thickened (Fig. 4). Significant destructive changes in the blood vessels were also seen.

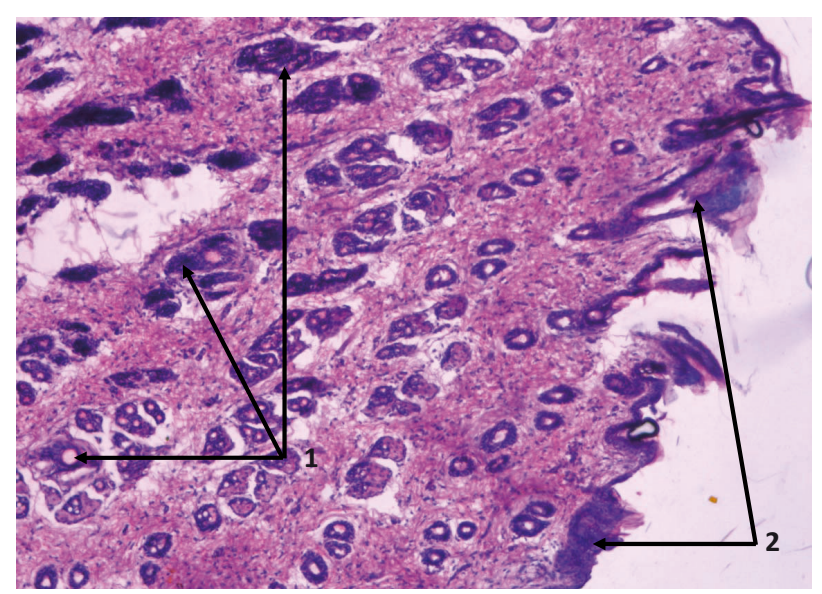

Figure 1. Skin of the lateral thigh surface of white rat after 2 weeks of nalbufin administration. Microphotograph. Stained with hematoxylin and eosin. The magnification: ob.x8, ey.x15

1 - a large number of hair follicles, 2 - expressed folds and normal epidermal thickness

These included endothelial desquamation into the lumen of blood vessels, smooth muscle hyperplasia of arteries and arterioles in the hypodermis, varicose veins and thrombosis in the hypodermis, as well as perivascular infiltrates. The wound defects were at different stages of the reparative process at the same time of the experiment, in the control animals. Therein, the inflammatory infiltration and vascular response were more evident; the proliferation and angiogenesis were more intense. Furthermore, the tissue was more infiltrated with leukocytes and macrophages, while more fibroblasts were detected. Regenerated epidermis was also differentiated, and a thin stratum corneum was being formed.

After 4 weeks of nalbufin administration, the wound healing slowed down, and was sometimes complicated by the formation of micro-abscesses. A large number of capillaries of granulation tissue, diffuse slightly marked acute inflammatory infiltrate, as well as degenerative and atrophic changes in the striated muscle fibers, were detected in the wound canals (Fig. 2). Of note, in some places there was swelling and fibrosis of the reticular dermis (Fig. 3). Only a few wound defects were closed and the regenerate complete. In most cases, the remodeling phase was not detected, some wound defects were filled with rough connective regenerate and the epidermis was thickened (Fig. 4). Significant destructive changes in the blood vessels were also seen. These included endothelial desquamation into the lumen of blood vessels, smooth muscle hyperplasia of arteries and arterioles in the hypodermis, varicose veins and thrombosis in the hypodermis, as well as perivascular infiltrates. The wound defects were at different stages of the reparative process at the same time of the experiment, in the control animals. Therein, the inflammatory infiltration and vascular response were more evident; the proliferation and angiogenesis were more intense. Furthermore, the tissue was more infiltrated with leukocytes and macrophages, while more fibroblasts were detected. Regenerated epidermis was also differentiated, and a thin stratum corneum was being formed. 


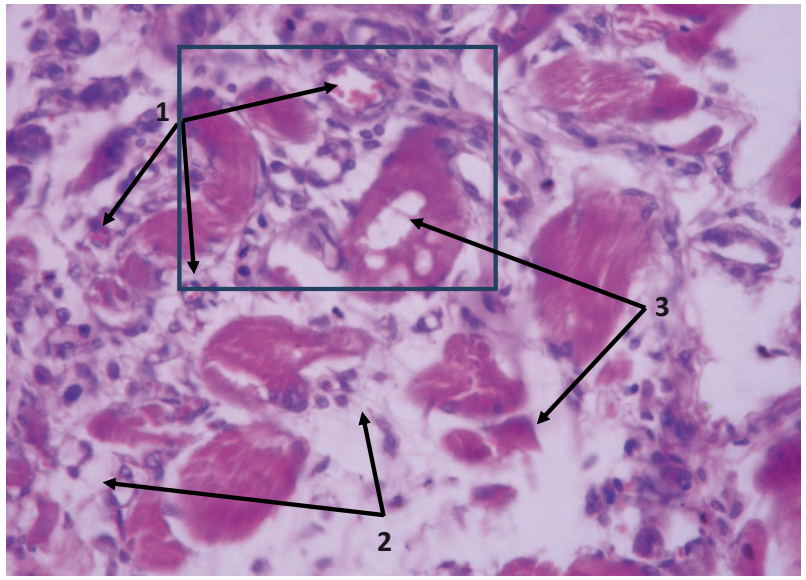

Figure 2. Skin of the lateral thigh surface of white rat after 4 weeks of nalbufin administration. Microphotograph. Stained with hematoxylin and eosin. The magnification: ob.x40, ey.x10

1 - a large number of capillaries in the granulation tissue in the wound canal, 2 - diffuse moderate expressed acute inflammatory infiltrate, 3 - degenerative and atrophic changes of striated fibers

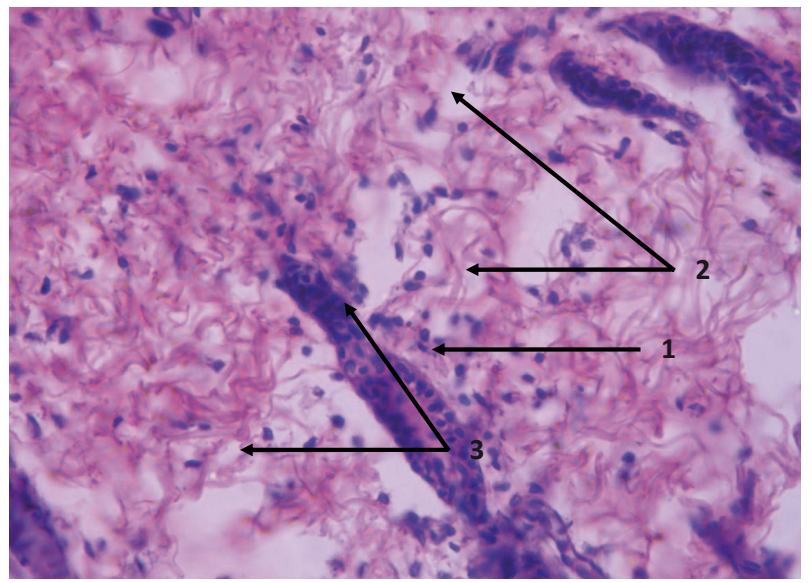

Figure 3. Skin of the lateral thigh surface of white rat after 4 weeks of nalbufin administration. Microphotograph. Stained with hematoxylin and eosin. The magnification: ob.x40, ey.x10

1 - hair bulb, 2 - moderately expressed round cell infiltration in the hypodermis, 3 - swelling of the reticular dermis

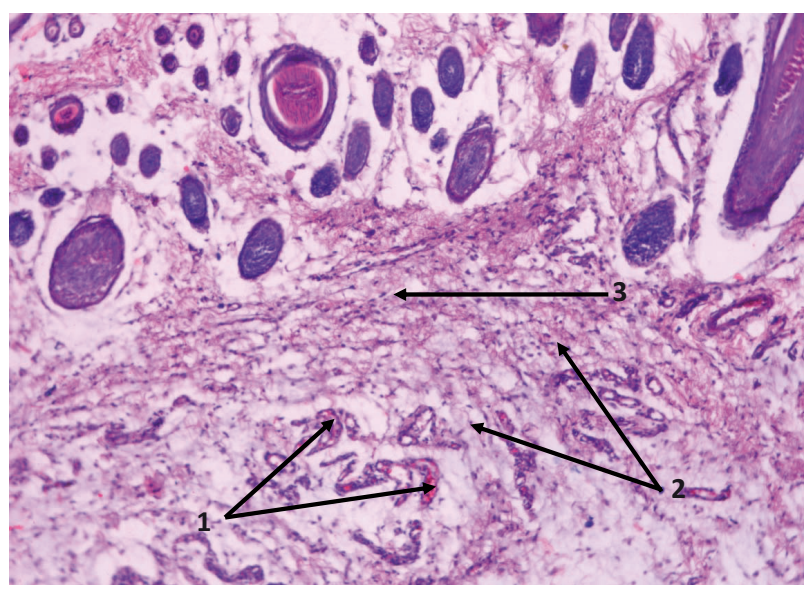

Figure 4. Skin of the lateral thigh surface of white rat after 4 weeks of nalbufin administration. Microphotograph. Stained with hematoxylin and eosin. The magnification: ob.x8, ey.x15

1 - a large number of newly formed capillaries of hypodermis, 2 - moderate diffuse inflammatory infiltrate (lymphocytes, macrophages, neutrophils) in the hypodermis, 3 - fibrosis of the reticular dermis
After 6 weeks of opioid administration, edema, infiltration with leukocytes and macrophages in wound defects during the phase of inflammation were detected (Fig. 5). Swelling with dissociation of interstitium of striated muscle, ruptures and different thickness of striated fibers were also seen. Moreover, the blood vessels were overfilled with blood. Furthermore, proliferation of smooth muscle cells in the vessel walls, aggregation, agglutination of red blood cells and thrombosis of venules were observed. In addition, marked endothelial proliferation, with the formation of an "endothelial hump", was seen in venules of the hypodermis, while the epidermis of the wound edges was thickened and the net of connective tissue fibers was dense inside the wound defects during the proliferation phase. Signs of hyperkeratosis, as well as perinuclear edema of basal and spinous epidermocytes were also observed in the regenerated epidermis. In addition, the inflammatory processes were intensified in some wound defects and excessive migration of neutrophil, granulocytes and macrophages was observed. Finally, a decrease in the number of the sebaceous glands, the hyperplasticity of sebaceous glands, and, mainly, a reduction in the number of hair follicles were detected (Fig. 6).

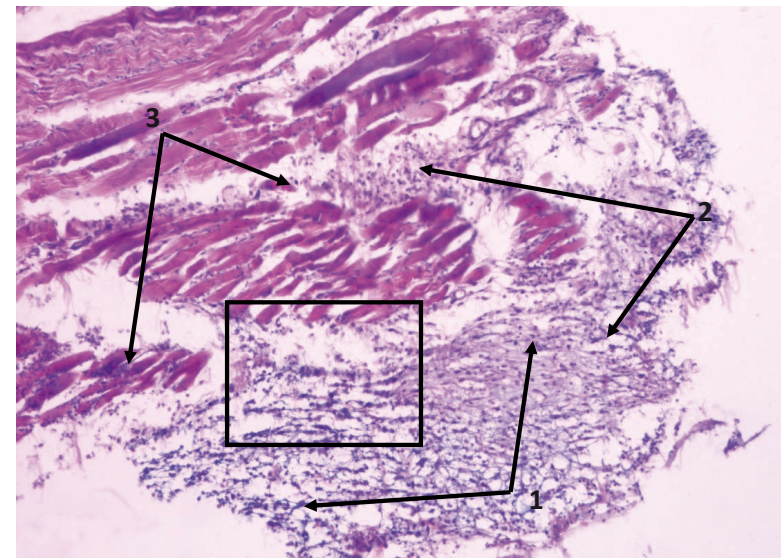

Figure 5. Skin of the lateral thigh surface of white rat after 6 weeks of nalbufin administration. Microphotograph. Stained with hematoxylin and eosin. The magnification: ob.x8, ey.x15

1 - swelling with dissociation of interstitium of striated muscle, 2 - polymorphonuclear cell infiltration in the wound canal, 3 - ruptures and various thickness of striated fibers

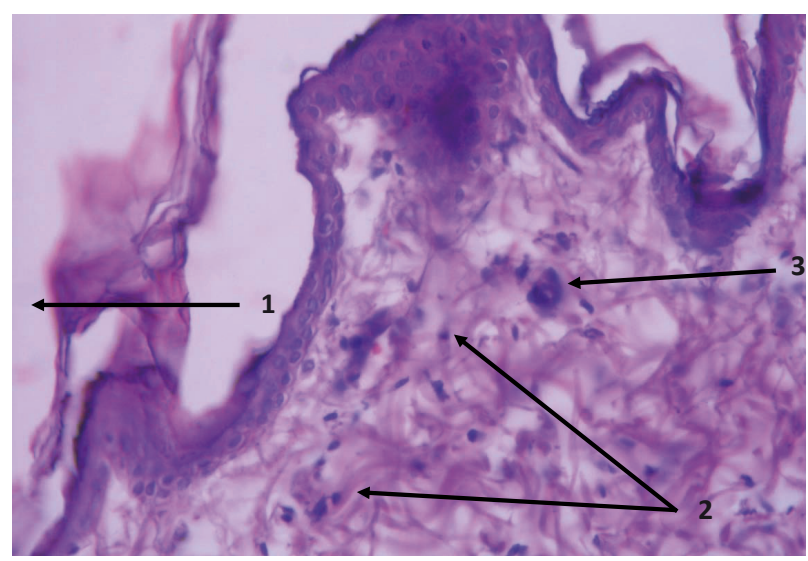

Figure 6. Skin of outer thigh of white rat after 6 weeks of nalbufin administration. Microphotograph. Stained with hematoxylin and eosin. The magnification: ob.x40, ey.x10

1 - hyperkeratosis of the epidermis, 2 - round cell infiltration in the hypodermis, 3 - atrophied hair bulb 
Regarding the control, after 6 weeks, all phases characteristic for the reparative process phase, starting from inflammation, proliferation, until complete healing of the wound defect, were observed.

\section{DISCUSSION}

The healing of wounds remains one of the most important scientific and practical problems in contemporary medicine $[4,7,9]$. Our results confirm that the healing of the skin (which is accompanied by phase changes in the structure of its structural elements - the epidermis, dermis and hypodermis), is affected by several endogenous and exogenous factors. In current literature, a number of works are devoted to the role of mast cells in the reparative process $[16,18]$. Mast cells are the source of biologically active substances such as histamine, heparin, platelet-activating factor, factors involved in the regulation of blood coagulation, factors of chemotaxis of neutrophils and eosinophils, metabolites of arachidonic acid, etc. The morphogenesis of mast cells is associated not only with the state of the vessel surface and deep vascular plexus, but also with the regulation of angiogenesis, cell proliferation of the fibroblastic series and epidermal keratinocytes, as well as with the cyclic changes in hair follicles and the remodelling of the dermis in the region of the wound defect.

Some authors state that in the course of wound healing, there are three distinct main periods. The first period is the melting of the necrotic mass and the cleaning of the wound defect (this comes about by way inflammation). The second period is the proliferation of connective tissue elements with the formation of granulation tissue. The third period is the fibrosing of the granulation tissue with scar formation and its epithelization $[4,10,14]$. Our work leads us to believing that this classification corresponds to modern ideas on the role of various cell components and intracellular interactions in the wound healing process. The healing of any wound occurs by way of involving the same cells and intercellular structures that contribute to similar general dynamics of the wound process, which is based on the typical processes such as inflammation, disturbance of microcirculation and tissue growth. However, types of cellular interactions vary in different phases of inflammation and regeneration. In addition, intercellular interactions are realized through cellular mediators and direct intercellular contacts. The role of these contacts is to transfer information via fibroblasts about cessation of proliferation [16,18].

Regeneration processes of the skin, according to many researchers, are the most expressive in the perivascular area of the capillary, while in remote areas of the capillary, biosynthetic activity of cells decreases, and their degenerative changes increase $[1,12,13]$. In tissues with active blood flow, the processes of proteolysis, phagocytosis, elimination of nonvital tissue and germs are more expressive. Thus, the researches aimed at improving blood flow to the tissues and the stimulation of angiogenesis through a variety of therapeutic effects are interesting. However, in the professional literature, there are only a little amount of data made available on the influence of opiates and opioids on wound healing. Yet, as indicated in our work, the myriad of problems that arise in the course of reparative processes, due to the presence of opioids, require further solution by means of adequate morphological and clinical studies. The results of our investigation show that opioid presence delays healing, hence the need to develop effective methods for the stimulation of the reparative processes.

\section{CONCLUSIONS}

The obtained experimental results show the negative impact of prolonged opioid administration on the reparative processes in the skin. After 4 weeks of nalbufin administration, the wound healing processes are slowed down and the formation of microabscesses comes about. After 6 weeks of opioid administration, destructive changes in the skin were detected. These resulted in the inability to complete the formation of the regenerate.

\section{REFERENCES}

1. Akhavani M.A. et al.: Angiogenesis and plastic surgery. Plast. Rec. Surg., 86(12), 1425, 2008

2. Assadian Q., Assadian A., Senekowitsch C.: Gas gangrene due to Clostridium perfringens in two injecting drug users in Vienna, Austria. Wien. Klin. Wschr., 116(7-8), 264, 2004.

3. Brown P.D., Ebright J.R.: Skin and soft tissue infections in injection drug users. Curr. Infect. Dis. Rep., 4(5), 415, 2002.

4. Criado P.R., Maruta C.W.: Histamine, histamine receptors and antihistamines: new concepts. An. Bras. Dermatol., 85(2), 195, 2010.

5. Currie B.J., Group.: A streptococcal infections of the skin: Molecular advances but limited therapeutic progress. Curr. Opin. Infect. Dis., 19, 132, 2006.

6. Dakal A.: Clinical signs of odontogenous suppirative and inflammatory diseases of maxillofacial are in drug-addicted patient, $7^{\text {th }}$ Congress of Baltic Association for Maxillofacial and Plastic Surgery, May 20-22, 71, 2010.

7. Datsenko B.M., Kutepova N.V., Borisenko V.B.: Transformation of collagens in infected wound with allotransplants. Clinical Surgery, 11, 16, 2011. (in Russian).

8. Friedman H., Pross S., Klein T.W.: Addictive drugs and their relationship with infectious diseases. FEMS Immunol. Med. Microbiol., 47(3), 330, 2006.

9. Gerych I.D. et al.: Liquid volumetric vulnerometry and criteria of vulnerometrical estimations of the wounds. Bulletin of the Ukrainian Medical Dental Academy, 9(1), 276, 2009.

10. Lipschitz G.V. Zvyahyntseva T.: Cell-cell interactions in the wound process. International Journal of Medical Scienses, 5(4), 120, 1999. (in Russian).

11. Onisko R.M. et al.: A method of modeling physical opioid dependence in rats, Patent: Danylo Halytsky Lviv National Medical University, Ukraine, u201207124; appl. 12.06.2012; publ. 10.01.2013, Bulletin number 1. (in Ukrainian).

12. Ozawa C.R. et al.: Microenvironmental VEGF concentration, not total dose, determines a threshold between normal and aber rant angiogenesis. J. Clin. Invest., 113(4), 516, 2004.

13. Pasichnyi D.A.: Macrovascular changes in skin wound in response to wound dermotension and cryotherapy. International Journal of Medical Scienses, 15(2), 68, 2009. (in Russian).

14. Sarkisov D.S. et al. (1990). Morphology of wound healing process. Medicine, Moscow; p.38-89. (in Russian).

15. Shestakova I.V., Yushchuk N.D., Tyshkevych O.L.: Myonecrosis in injecting drug users (clinical observation). Therapeutic Archives, 82(11), 37, 2010. (in Russian).

16. Theoharides C.T., Singh K.L., Boucher W.: Cor ticotropin-releasing hormone induces skin mast cell degranulation and increased vascular permeability, a possible explanation for its proinflammatory effects. Endocrinology, 139(1), 403, 2005 
17. Tymofiev A.A., Dakal A.V.: The use of modern drugs for the local treatment of purulent wounds in drug addicted patients with odontogenic abscesses. Modern dentistry, 4(44), 95, 2008. (in Russian).
18. Weller K. et al.: Mast cells are required for normal healing of skin wounds in mice. The FASEB Journal, E1, 2006. 